\title{
Comparação entre as técnicas de mensuração da pressão arterial em um e em dois tempos
}

\author{
Comparison of the techniques of measuring blood pressure in one and in two steps
}

Comparación entre las técnicas de medición de presión arterial en uno y en dos tiempos

\author{
Cristiane Crisp Martins Ribeiro', José Luiz Tatagiba Lamas" \\ ' Prefeitura Municipal de Campinas. Campinas-SP, Brasil. \\ "Universidade Estadual de Campinas, Faculdade de Ciências Médicas, \\ Departamento de Enfermagem. Campinas-SP, Brasil.
}

Submissão: 09-05-2011 Aprovação: 15-10-2012

\begin{abstract}
RESUMO
Medir a pressão arterial é passo inicial e indispensável para um adequado diagnóstico de hipertensão, considerando que os níveis de pressão representam importante marcador de risco para doenças cardiovasculares. Medir a pressão, entretanto, pode resultar em conclusões inapropriadas se normas técnicas básicas não forem obedecidas. Este estudo comparou o uso da técnica de mensuração da pressão arterial em um tempo com a técnica em dois tempos, recomendada pela American Heart Association em 2005. Foram realizadas medidas pelas duas técnicas em quarenta indivíduos adultos, utilizando-se como equipamentos manômetro de mercúrio e aparelho digital. Pela análise dos dados concluiu-se que a técnica em um tempo apresentou resultados similares àqueles obtidos com a técnica em dois tempos.
\end{abstract}

Descritores: Pressão Arterial; Determinação da Pressão Arterial; Hipertensão.

\begin{abstract}
Measuring blood pressure is an essential and initial step in seeking a proper diagnosis of hypertension, once blood pressure levels are an important risk marker for cardiovascular disease. Measuring blood pressure, however, can result in inappropriate conclusions if basic technical standards are not obeyed. This study compared the use of the technique for measuring blood pressure in one step with the technique in two steps, recommended by the American Heart Association in 2005. Measurements were made by both techniques in fourteen adults, using mercury sphygmomanometer and a digital device. Through analysis of the data it was concluded that the technique in one step showed similar results to those obtained with the technique in two steps.
\end{abstract}

Key words: Blood Pressure; Blood Pressure Determination; Hypertension.

\section{RESUMEN}

Medir la presión arterial es un paso inicial e indispensable en la búsqueda de un adecuado diagnóstico de hipertensión, ya que los niveles de presión arterial representan un importante marcador de riesgo para enfermedades cardiovasculares. Sin embargo, medir la presión puede resultar en conclusiones inapropiadas si las normas técnicas básicas no son obedecidas. En este estudio comparamos el uso de las técnicas de medición de la presión arterial a un tiempo con la técnica a dos tiempos, recomendada por la American Heart Association en 2005. Hemos realizado mediciones por las dos técnicas en 40 individuos adultos, utilizando como equipamiento tensiómetro de mercurio y dispositivos digitales. Por medio del análisis de los datos se concluyó que la medición a un tiempo mostró resultados similares a los obtenidos con la técnica a dos tiempos.

Palabras clave: Presión Sanguínea; Determinación de la Presión Sanguínea; Hipertensión. 


\section{INTRODUÇÃO}

A medida da pressão arterial (PA) pelo método indireto com técnica auscultatória é um procedimento relativamente simples, confiável e muito utilizado na prática clínica para se avaliar os níveis da PA.

Esse procedimento requer dois equipamentos, um estetoscópio e um esfigmomanômetro, este último composto pelo manguito, manômetro, sistema de válvulas, pera e tubos de borracha. A inflação do manguito oclui o fluxo arterial, sua deflação leva à redução da pressão do sistema, até que a pressão de pico gerada pela contração do ventrículo esquerdo impulsiona o sangue num fluxo intermitente pelo leito da artéria, produzindo os sons de Korotkoff. A medida ocorre por meio da oclusão arterial pela inflação do manguito, relacionando a ausculta dos sons com o valor registrado na coluna de mercúrio. Esses ruídos são identificados por fases, cujo início e fim são utilizados para avaliar a pressão arterial sistólica (fase I) e a pressão arterial diastólica (fase $\mathrm{V})^{(1)}$.

Decorrido cerca de um século da descoberta dos sons de Korotkoff, o conhecimento na área da medida da pressão, no diagnóstico precoce e tratamento da hipertensão arterial e na avaliação dos fatores de risco para moléstias cardiovasculares permanece com lacunas e é fonte de pesquisa em todo mun$\mathrm{do}^{(2)}$. O procedimento fundamental para a realização dessas pesquisas e para a evolução do conhecimento na área é a medida da PA. Apesar de extremamente difundida e rotineiramente utilizada, esta determinação, principalmente pelo método auscultatório, ainda é realizada, frequentemente, sem a observação das recomendações básicas para evitar erro nas aferições $s^{(3-5)}$.

Visando a padronização da medida da pressão arterial, entidades internacionais como a American Heart Association ${ }^{(3)}$ e o VII Joint National Committee on Prevention, Detection, Evaluation, and Treatment of High Blood Pressure ${ }^{(6)}$ tecem recomendações a respeito de todos os cuidados necessários para a realização da medida correta da pressão arterial. Do mesmo modo, sociedades científicas brasileiras elaboram em conjunto suas recomendações para a abordagem dos hipertensos, que incluem a técnica de medida da $\mathrm{PA}^{(1)}$.

Atualmente, as VI Diretrizes Brasileiras de Hipertensão(1) preconizam para a medida da pressão arterial a técnica em dois tempos. A importância da realização desta técnica consiste em proporcionar, através do método palpatório, uma aproximação preliminar necessária da pressão arterial sistólica e assegurar um nível adequado de inflação antes que a medição auscultatória seja feita. Ela é particularmente útil porque evita o enchimento insuficiente do manguito em pacientes com hiato auscultatório e a hiperinsuflação naqueles com pressão arterial muito baixa ${ }^{(7)}$. Para sua execução é preciso realizar o preparo do paciente e a medida em si.

Primeiramente deve-se explicar o procedimento ao paciente; promover o repouso de pelo menos 5 minutos em ambiente calmo; permitir que ele use o banheiro, verificar se não praticou exercícios físicos nos últimos 60 minutos, não ingeriu bebidas alcoólicas, café ou alimentos e não fumou nos 30 minutos anteriores; orientá-lo para, durante o procedimento, não falar, manter as pernas descruzadas, os pés apoiados no chão, o dorso recostado na cadeira e permanecer relaxado. Deve-se retirar a roupa do braço onde será feita a medida e posicionar o braço do paciente na altura do coração (nível do ponto médio do esterno ou $4^{\circ}$ espaço intercostal), apoiado, com a palma da mão voltada para cima e cotovelo ligeiramente fletido ${ }^{(1,3)}$.

Após esse preparo, a medida em dois tempos preconiza a estimativa palpatória da pressão sistólica (primeiro tempo) e a determinação auscultatória da PA (segundo tempo). No primeiro tempo deve-se medir a circunferência do braço do paciente e selecionar manguito de tamanho adequado; colocar o manguito sem deixar folgas 2 a $3 \mathrm{~cm}$ acima da fossa cubital; centralizar o meio da parte compressiva do manguito sobre a artéria braquial; estimar o nível da pressão sistólica (palpar o pulso radial e inflar o manguito até o seu desaparecimento, desinflando rapidamente; o reaparecimento do pulso corresponderá à pressão sistólica). Após um minuto procede-se ao segundo tempo, que consiste em palpar a artéria braquial na fossa cubital e colocar a campânula do estetoscópio sem compressão excessiva; inflar rapidamente até ultrapassar 20 a 30 mmHg o nível estimado da pressão sistólica; proceder à deflação lentamente (velocidade de 2 a $4 \mathrm{mmHg}$ por segundo); determinar a pressão sistólica na ausculta do primeiro som (fase I de Korotkoff), que é um som fraco seguido de batidas regulares, e, após, aumentar ligeiramente a velocidade de deflação; determinar a pressão diastólica no desaparecimento do som (fase $\mathrm{V}$ de Korotkoff); auscultar cerca de 20 a $30 \mathrm{mmHg}$ abaixo do último som para confirmar o seu desaparecimento e depois proceder à deflação rápida e completa; se os batimentos persistirem até o nível zero, determinar a pressão diastólica no abafamento dos sons (fase IV de Korotkoff) e anotar os valores da sistólica/diastólica ${ }^{(1,3)}$.

O que se observa na prática, afora diversos erros cometidos, é o uso da técnica em um tempo, que, normalmente, não realiza a estimativa palpatória da pressão sistólica. Nela o profissional infla o manguito até um ponto decidido aleatoriamente por ele mesmo, e em seguida o desinsufla fazendo a ausculta dos sons de Korotkoff. A justificativa apresentada por esses profissionais é a economia de tempo que essa técnica permite.

Verifica-se que, a cada uma das recomendações, novos aspectos relacionados ao observador, equipamento, paciente, ambiente e técnica são discutidos com o intuito de afastar possibilidades de erros, que comprometam a fidedignidade da medida de pressão arterial e, conseqüentemente, do diagnóstico da hipertensão ${ }^{(1)}$.

Em relação ao aparelho, podemos citar a má conservação, os vazamentos, a não calibração, a inadequação do manguito à circunferência do braço. Se o manguito for muito largo, os níveis de pressão obtidos serão mais baixos e se o manguito for muito estreito, os níveis serão falsamente elevados. Para evitar esse tipo de erro, é recomendado usar manguitos adequados à circunferência do braço ${ }^{(1,8)}$. Ao observador podemos atribuir fatores tais como a hipoacusia, a má colocação do manguito, a inflação excessiva da bolsa, a deflação muito rápida, a compressão da artéria braquial pelo estetoscópio, o uso 
incorreto do método de mensuração e outros ${ }^{(3)}$. A obesidade, a presença de arritmias e a hipertensão do avental branco são causa de algumas flutuações atribuídas ao paciente ${ }^{(9)}$. Quanto ao ambiente, podemos citar a temperatura da sala, o barulho de fundo, vindo de outros locais, e a luminosidade ${ }^{(3,9)}$.

A preocupação das sociedades científicas com esse procedimento não é desprovida de sentido, já que se trata de um dos procedimentos de saúde mais executados em todo o mundo, por profissionais de diversas formações. Dentre elas destaca-se a categoria da enfermagem, a qual desempenha papel importante na medida da pressão arterial, seja na detecção precoce da doença ou no controle e acompanhamento dos hipertensos, bem como na orientação destas pessoas. Sabe-se que os valores obtidos pela medida da pressão arterial são um importante indicador de saúde e apresentam correlação direta com a morbidade cardiovascular ${ }^{(10)}$.

Considerando, então, a magnitude da hipertensão arterial e a importância da detecção precoce da doença, a comparação da técnica da mensuração da pressão arterial em um tempo em relação à técnica em dois tempos pode ser um fator simplificador. Ou seja, a confirmação de que as duas técnicas podem proporcionar a obtenção de valores similares com o mesmo grau de confiabilidade poderia resultar em economia de esforços e recursos necessários para a capacitação do observador, além de benefícios para a população.

\section{OBJETIVO}

Comparar valores da pressão arterial obtidos pelas técnicas de mensuração em um tempo e em dois tempos, com manômetro de mercúrio, nos membros superiores.

\section{MATERIAL E MÉTODO}

Trata-se de um estudo observacional transversal. O estudo foi realizado nas Unidades de Internação e nos Ambulatórios de um hospital de ensino do interior de São Paulo. Os sujeitos do estudo foram pacientes atendidos nos Ambulatórios e nas Unidades de Internação do hospital.

A amostra foi composta por indivíduos acima de 18 anos, cuja circunferência braquial, medida no ponto médio do braço, variava de 31 a 33 centímetros. Esse valor foi tomado como referência de acordo com as recomendações da American Heart Association ${ }^{(11)}$, que preconiza uma relação de 0,38 entre a largura do manguito e circunferência do braço (nesses sujeitos o manguito de largura correta é o de $12 \mathrm{~cm}$ ). Essa relação tem sido referida como sendo de 0,4 em publicações mais recentes ${ }^{(3)}$. Os sujeitos foram selecionados por amostragem casual. Foram excluídos todos aqueles que apresentaram contraindicações para a medida da pressão arterial no braço (por exemplo: indivíduos em tratamento de hemodiálise e indivíduos que passaram por tratamento cirúrgico de retirada de redes linfáticas em MMSS) e indivíduos cuja diferença de pressão entre os dois braços foi superior a $4 \mathrm{mmHg}$, tanto para mais quanto para menos, tanto na pressão sistólica quanto na diastólica. Isso atende a recomendação feita por $\mathrm{O}^{\prime}$ Brien et. al. ${ }^{(12)}$. Apesar do objetivo deste trabalho não ser a validação de um instrumento automático, consideramos oportuno trabalhar com medidas muito próximas entre os dois braços, afim de que as possíveis diferenças estatísticas encontradas não pudessem ser atribuídas a diferenças entre os braços, mas sim entre as técnicas utilizadas.

Definiu-se uma amostra de 40 indivíduos para o estudo e, após análise pelo Software Minitab, verificou-se que a mesma tinha um poder de teste igual a 0,97, assegurando a sua validade e significância.

As variáveis analisadas foram os valores de pressão arterial sistólico e diastólico, obtidos por diferentes métodos e equipamentos.

Para a coleta de dados foram utilizados: um manômetro de mercúrio, um aparelho de pressão digital Omron (Modelo: HEM-714INT), ambos calibrados um mês antes do início da coleta de dados, um estetoscópio Littmann (tamanho adulto), um manguito de $12 \mathrm{~cm}$ de largura e almofadas para ajuste da posição do braço.

Os dados foram coletados por uma aluna do último semestre do Curso de Graduação em Enfermagem de uma universidade do interior paulista, devidamente capacitada na técnica de medida da PA em um tempo e em dois tempos, bem como na utilização do aparelho digital.

A coleta de dados foi realizada em salas de atendimento dos ambulatórios e quartos de internação, locais bem iluminados e livres de excesso de ruído, após 5 minutos de repouso na posição sentada, sem o indivíduo ter previamente ingerido café ou utilizado tabaco, com o braço apoiado no nível do coração.

Foram realizadas cinco medidas de pressão arterial em cada sujeito. A primeira foi feita com o aparelho de pressão digital validado em um dos braços, escolhido por sorteio, e a segunda, utilizando o mesmo aparelho no outro braço. Essas duas medidas foram realizadas para assegurar que a pressão arterial nos dois braços era igual ou diferia em até $4 \mathrm{mmHg}^{(12)}$ para mais ou para menos, tanto na pressão sistólica quanto na diastólica, o que assegurou a comparação entre os dois braços nas medidas subseqüentes.

A terceira medida foi feita simultaneamente nos dois braços, utilizando o aparelho digital em um deles e o manômetro de mercúrio no outro. O aparelho digital foi utilizado para, primeiramente, possibilitar medidas simultâneas pelo único observador e, segundo, para demonstrar a estabilidade da pressão arterial nos diferentes momentos, o que significaria que alterações de valores observadas seriam consequência da mudança da técnica. Além disso, optou-se por escolher um aparelho digital previamente validado segundo as recomendações da European Society of Hypertension (ESH) ${ }^{(13)}$.

Para a medida com o manômetro de mercúrio foi escolhido por sorteio uma das técnicas de medida da pressão arterial indireta: em um tempo ou em dois tempos, seguindo o protocolo da American Heart Association. Essa medida tinha o objetivo de possibilitar ao paciente conhecer as sensações provocadas pela inflação e deflação do manguito ${ }^{(14)}$ e não foi considerada quando da análise dos dados. A quarta medida foi exatamente igual à terceira, porém seus resultados foram 
utilizados para análise. A quinta e última medida também foi como a terceira, modificando apenas a técnica utilizada com o manômetro de mercúrio, ou seja, se na terceira e quarta medidas utilizou-se a técnica em um tempo, na quinta foi utilizada a técnica em dois tempos e vice-versa. As medidas realizadas com os próximos participantes tiveram sua ordem alternada a partir da terceira medida.

A medida em um tempo foi realizada obedecendo-se aos seguintes passos: medir a circunferência do braço do paciente e selecionar manguito de tamanho adequado; colocar o manguito sem deixar folgas 2 a $3 \mathrm{~cm}$ acima da fossa cubital; centralizar o meio da parte compressiva do manguito sobre a artéria braquial; palpar o pulso braquial e inflar o manguito até 20 a $30 \mathrm{mmHg}$ acima do ponto correspondente ao seu desaparecimento (nível estimado da pressão sistólica); colocar a campânula do estetoscópio sobre o ponto em que se palpou a artéria braquial, sem realizar compressão excessiva; proceder à deflação lentamente (velocidade de 2 a $4 \mathrm{mmHg}$ por segundo); determinar a pressão sistólica na ausculta do primeiro som (fase I de Korotkoff), que é um som fraco seguido de batidas regulares, e, após, aumentar ligeiramente a velocidade de deflação; determinar a pressão diastólica no desaparecimento do som (fase $\mathrm{V}$ de Korotkoff); auscultar cerca de 20 a $30 \mathrm{mmHg}$ abaixo do último som para confirmar o seu desaparecimento e depois proceder à deflação rápida e completa; se os batimentos persistirem até o nível zero, determinar a pressão diastólica no abafamento dos sons (fase IV de Korotkoff) e anotar os valores da sistólica/diastólica ${ }^{(1,3)}$.

Foram utilizados para análise estatística apenas os dados da quarta e quinta medidas. Para a análise descritiva das variáveis sexo e faixa etária foi utilizado o Software Excel.
Os valores de pressão arterial foram analisados utilizando teste $\mathbf{t}$ pareado e o teste de correlação de Pearson. Foram considerados significativos valores de $p<0,05$. A análise foi feita usando o Software estatístico Minitab e o programa SPSS para Windows versão 16.0.

O protocolo de pesquisa foi avaliado e aprovado pelo Comitê de Ética em Pesquisa local, parecer No 547/2009, em consonância com as determinações do CONEP - Resolução 196/96. A coleta de dados foi iniciada após a autorização do hospital para realização do estudo.

\section{RESULTADOS}

Os dados do presente estudo indicaram que $75 \%$ da amostra estudada eram do sexo feminino e $25 \%$ do masculino. Destes 40 indivíduos estudados, a média de idade foi de 36,3 anos. Os valores de pressão arterial obtidos com as diferentes técnicas e os diferentes aparelhos estão apresentados na Tabela 1.

Não foi observada diferença estatística significativa ( $p>$ 0,05 - teste $\boldsymbol{t}$ pareado) para os valores de pressão arterial sistólica e diastólica, o que representa uma similaridade de valores de pressão arterial, obtidos tanto pelos aparelhos quanto pelas técnicas diferentes.

Os dados obtidos com o aparelho digital demonstram que a pressão arterial se manteve estável durante a realização de todas as medidas do estudo.

As Figuras 1 e 2 mostram os valores de pressão medidos pelas duas técnicas, com o manômetro de mercúrio, em cada indivíduo. Neles as duas técnicas foram nomeadas como tratamento. Para efeito de cálculo, os valores da pressão arterial foram separados em valor sistólico e diastólico e comparados entre as duas formas de medição, em um (1T) e em dois tempos

Tabela 1 - Valores da média, desvio padrão, mediana, mínimo e máximo da pressão arterial sistólica (PAS) e diastólica (PAD) de acordo com o aparelho utilizado e a técnica realizada. Campinas-SP, 2009.

\begin{tabular}{|c|c|c|c|c|c|c|c|c|c|c|}
\hline \multirow{2}{*}{ Aparelho/ Técnica } & \multicolumn{2}{|c|}{ Média (mmHg) } & \multicolumn{2}{|c|}{$\begin{array}{l}\text { Desvio Padrão } \\
\quad(\mathrm{mmHg})\end{array}$} & \multicolumn{2}{|c|}{ Mediana $(\mathrm{mmHg})$} & \multicolumn{2}{|c|}{ Mínimo $(\mathrm{mmHg})$} & \multicolumn{2}{|c|}{ Máximo $(\mathrm{mmHg})$} \\
\hline & PAS & PAD & PAS & PAD & PAS & PAD & PAS & PAD & PAS & PAD \\
\hline $\begin{array}{l}\text { Digital (simultânea à medida } \\
\text { em um tempo) }\end{array}$ & 116,58 & 71,05 & 18,18 & 11,10 & 112 & 71 & 89 & 49 & 179 & 97 \\
\hline Mercúrio/ 1 tempo & 115,80 & 73,15 & 19,48 & 10,65 & 111 & 73 & 82 & 48 & 178 & 94 \\
\hline $\begin{array}{l}\text { Digital (simultânea à medida } \\
\text { em dois tempos) }\end{array}$ & 116,58 & 72,55 & 19,44 & 11,22 & 110,5 & 73,5 & 93 & 49 & 178 & 96 \\
\hline Mercúrio/ 2 tempos & 115,33 & 72,35 & 19,86 & 10,95 & 110 & 74 & 88 & 46 & 178 & 92 \\
\hline
\end{tabular}




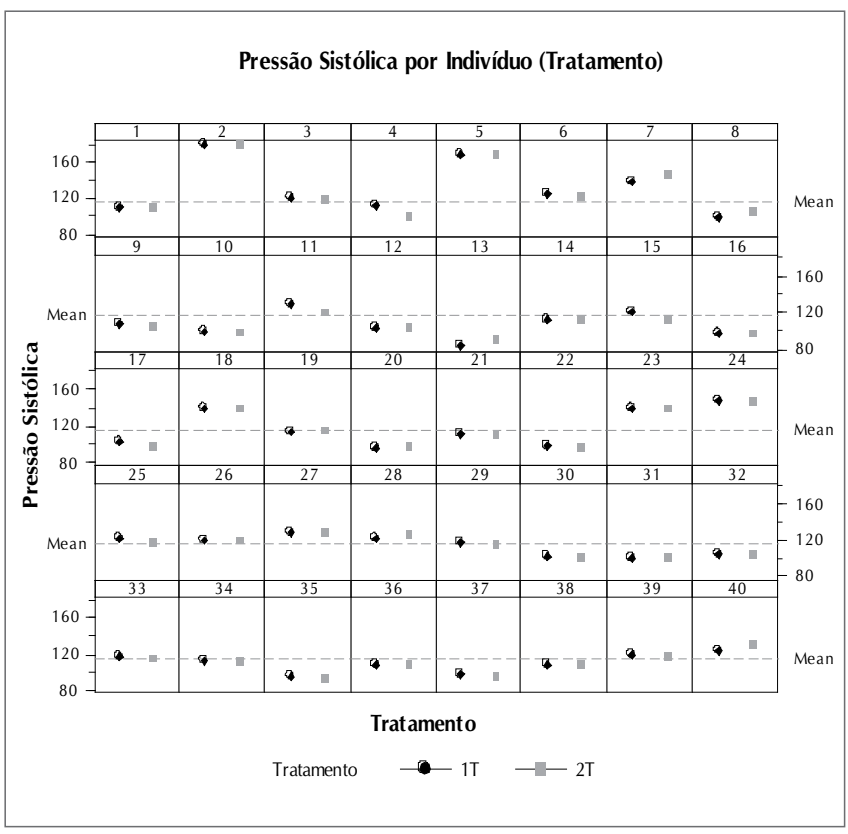

Figura 1 - Valores de pressão sistólica por indivíduo para as técnicas em um e em dois tempos. Campinas-SP, 2009.

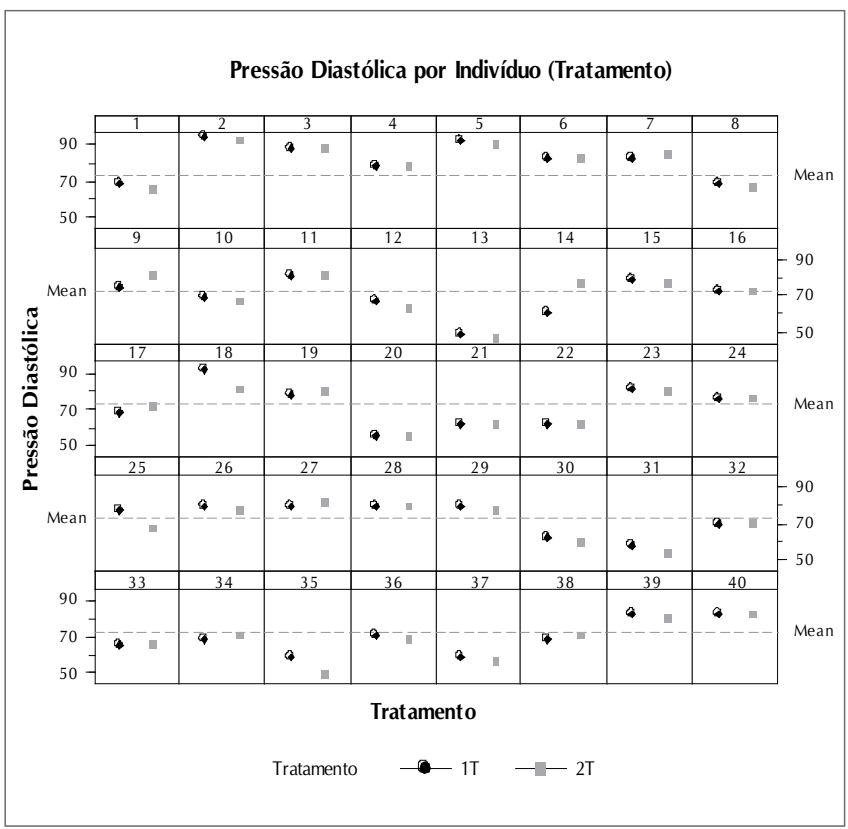

Figura 2 - Valores de pressão diastólica por indivíduo para as técnicas em um e em dois tempos. Campinas-SP, 2009.
(2T). Pode-se perceber que a distância vertical entre os dois tratamentos para a maioria dos indivíduos permanece nula, ou próxima a zero, exceto para o indivíduo número 14, especificamente no que se refere à pressão diastólica (Figura 2).

A Tabela 2 mostra o coeficiente de correlação (r) encontrado a partir dos valores de pressão arterial sistólica e diastólica para ambas as técnicas, assim como o nível de significância do teste de correlação de Pearson (p-valor).

Foi observado alto grau de correlação entre as técnicas utilizadas (um ou dois tempos) atingindo significância estatística. Isso reforça a conclusão de que as mesmas propiciaram a obtenção de valores equivalentes.

\section{DISCUSSÃO}

Este trabalho teve como objetivo comparar duas técnicas de mensuração indireta da pressão arterial, levando em consideração que a técnica em dois tempos é recomendada por entidades nacionais ${ }^{(1)}$ e internacionais ${ }^{(3)}$ e a técnica em um tempo é utilizada rotineiramente por profissionais da área da saúde nos diversos serviços de saúde.

Observa-se na literatura, pelos inúmeros estudos publicados, a grande preocupação dos pesquisadores em identificar se os profissionais da saúde têm realizado este procedimento de modo adequado, principalmente pelo fato de estar diretamente relacionado com a avaliação de um parâmetro vital(4) $^{(4)}$. Um estudo realizado por Rabello et. al. ${ }^{(15)}$ avaliou o conhecimento teórico e prático de auxiliares de enfermagem, médicos e enfermeiros, por meio de 15 questões de múltipla escolha e pela observação do procedimento da medida da pressão arterial, e concluiu que o conhecimento de todos os profissionais foi insatisfatório, característica também evidenciada no trabalho de Almeida ${ }^{(16)}$.

Observa-se que tem sido crescente também o número de estudos preocupados em descrever os erros mais freqüentes na medida da pressão arterial. Ao avaliarem as técnicas de medida e os erros cometidos por diferentes profissionais na medida rotineira da pressão arterial, autores ${ }^{(4-5)}$ evidenciaram $^{-1}$ que, em todas as categorias profissionais estudadas, existe a necessidade de melhor desempenho nessa técnica. Maior atenção deve ser dada às fontes de erro (ambiente, indivíduo, observador e instrumento), intimamente ligadas aos valores obtidos. Ressalta-se, por exemplo, que a hipo e a hiperestimação da pressão arterial sao fenômenos que ocorrem com frequência e que as suas consequências na falta de diagnóstico e tratamento da hipertensão arterial as tornam um grave problema $^{(2)}$.

Tabela 2 - Coeficientes de correlação de Pearson ( $r$ ) e nível de significância ( $p$-valor) entre os valores de pressão obtidos pelas técnicas em um tempo (1T) e dois tempos (2T), para as pressões sistólica e diastólica. Campinas-SP, 2009.

\begin{tabular}{cccc}
\hline Pressão arterial & R & (p-valor) & $<0,01$ \\
\hline Sistólica & $1 \mathrm{~T} \times 2 \mathrm{~T}$ & 0,98 & $<0,01$ \\
Diastólica & $1 \mathrm{~T} \times 2 \mathrm{~T}$ & 0,92 &
\end{tabular}


Ao estudarem o conhecimento relacionado à medida da pressão arterial, dois autores ${ }^{(15-16)}$ observaram um preocupante desconhecimento dos aspectos teóricos da técnica. Este fato também foi observado por outros autores ${ }^{(2)}$, que concluíram em seu trabaIho que o conhecimento de muitos profissionais sobre a medida da pressão é deficitário, o procedimento é executado sem bases científicas e inexistem programas de educação permanente.

Pensando que a adequação das condutas terapêuticas e a validade das inferências epidemiológicas dependem essencialmente da acurácia dos métodos e procedimentos de aferição da pressão arterial, a minimização de erros de aferição é crucial para reduzir a ocorrência de diagnósticos equivocados de hipertensão arterial e assegurar um tratamento adequado aos pacientes hipertensos.

Por esse motivo, entidades especializadas em cardiologia se reúnem em eventos mundiais para discutir e estabelecer normas técnicas de mensuração da pressão arterial, a serem seguidas pelos diferentes profissionais de saúde. As normas, baseadas em estudos sobre hipertensão e pressão arterial, asseguram uma fidedignidade dos valores obtidos desde que a mensuração siga todos os passos pré-estabelecidos.

Apesar disso, sabemos que nem todos os profissionais da área realizam a medida da pressão arterial conforme o recomendado. Nos diversos serviços de saúde, a técnica em dois tempos dá lugar à técnica em um tempo, que, por ser realizada em menos etapas, acelera o processo de mensuração da pressão, mas por outro lado, pode estar mais sujeita a erros. Caso a técnica em um tempo não inclua a estimativa palpatória da pressão sistólica, o observador pode, naqueles pacientes com hiato auscultatório, estar sujeito a não identificar corretamente durante a ausculta o som correspondente à pressão sistólica, ou então superestimar a pressão diastólica.

Aponta-se como fonte de erro na medida da pressão arterial os próprios cursos de graduação, que podem não estar preparando adequadamente os profissionais para esse procedimento específico ${ }^{(4)}$, propondo, assim, a necessidade urgente de se desenvolver estratégias de ensino e aprendizagem com este foco, como cursos de atualização, investindo na educação continuada e inserindo conteúdos específicos nos cursos de graduação e pós-graduação. Avaliou-se o conhecimento sobre hipertensão e seu tratamento com a equipe de enfermagem, antes e após onze intervenções educativas, concluindo-se que as mesmas foram eficazes e que devem ser implementadas junto à equipe de enfermagem, considerando que elas podem influenciar no aprimoramento da assistência às pessoas hipertensas ${ }^{(17)}$.

Foi observado que ainda não existem estudos na literatura comparando as duas técnicas, a fim de avaliar se o uso da técnica não recomendada também é uma fonte de erro, relacionada principalmente ao observador.

Este estudo mostrou um alto grau de correlação entre as técnicas, tanto para a pressão sistólica $(r=0,98)$, quanto para a diastólica ( $r=0,92)$, e um nível de significância inferior a 0,01, demonstrando que não houve diferenças entre os valores obtidos por estas duas técnicas, conforme aplicadas nesta pesquisa.

Com os resultados deste estudo podemos concluir que o uso da técnica em um tempo não contribuiu como fonte de erro na medida da PA para essa amostra populacional, ou seja, não forneceu dados imprecisos para diagnósticos de hipertensão e, dessa forma, sua aplicação não privaria os pacientes de um tratamento adequado, desde que inclua a estimativa palpatória da pressão sistólica.

Assim, podem-se economizar esforços e recursos que visem capacitar os profissionais de saúde na execução da técnica em dois tempos, direcionando esses mesmos esforços e recursos para diminuir a ocorrência dos demais erros observados. É necessário estimular outros estudos que avaliem a possibilidade de se recomendar a técnica em um tempo antes que a mesma possa ser adotada com segurança.

\section{CONCLUSÃO}

Concluiu-se com a análise dos dados que a medida indireta da pressão arterial pela técnica em um tempo, com a inclusão da estimativa palpatória da pressão sistólica, não difere da técnica recomendada mundialmente, em dois tempos. Além disso, ambas as técnicas fornecem valores equivalentes aos obtidos pelo aparelho digital validado. Esse achado faz com que os esforços e os recursos necessários para a capacitação dos profissionais de saúde na execução da técnica recomendada sejam reduzidos e estimula estudos que avaliem a possibilidade de também se recomendar a técnica em um tempo. Entretanto, não é possível generalizar esses resultados, considerando que foi estudada uma população específica, sendo necessários mais estudos relacionados ao tema antes que essa técnica possa ser amplamente recomendada.

\section{REFERÊNCIAS}

1. Sociedade Brasileira de Hipertensão. DBH (VI Diretrizes Brasileiras de Hipertensão). Rev Hipertens 2010;13(1):08-66.

2. Arcuri EAM; Araújo TL; Veiga EV; Oliveira SMJV; Lamas JLT; Santos JLF. Medida da pressão arterial e a produção científica de enfermeiros brasileiros. Rev Esc Enferm USP 2007;41(2):292-8.

3. Pickering TG, Hall JE, Appel LJ, Falkner BE, Graves J, Hill $M N$, et al. Recommendations for blood pressure measurement in humans and experimental animals: part 1:
Blood Pressure Measurement in humans. A Statement for professionals from the subcommittee of professional and public education of the American heart Association Council on High blood pressure research. Hypertens 2005;45(1):142-61.

4. Veiga EV, Nogueira MS, Cárnio EC, Marques S, Lavrador MAS, de Moraes SA, Souza LAC, Lima NKC, Nobre F. Avaliação de técnicas da medida da pressão arterial pelos profissionais de saúde. Arq Bras Cardiol 2003;80(1):83-9. 
5. Lamas JLT, Berno CBF, Takeiti GM. Erros cometidos por profissionais de enfermagem na medida rotineira da pressão arterial. Rev Paul Enferm 2003;22(2):141-8.

6. Chobanian AV, Bakris GL, Black HR, Cushman WC, Green LA, Izzo JL, Jones DW, Materson BJ, Oparil S, Wright JT, Roccella EJ, the National High Blood Pressure Education Program Coordinating Committee. The Seventh Report of the Joint National Committee on Prevention, Detection, Evaluation and treatment of high Blood Pressure. NIH Publication $n^{\circ} 03-5233$. Hypertens 2003;42(6):1206-52.

7. Perloff D, Grim C, Flack J, Frohlich ED, Hill M, McDonald M, Morgenstern BZ. Human blood pressure determination by sphygmomanometry. Circul 1993;88:2460-70.

8. Lamas JLT, Arcuri EAM, Brito CM, Cruz KCT. Registros intra-arteriais da pressão versus registros indiretos em função da largura do manguito. Rev Gaúch Enferm 2006;27(4):599-609.

9. Schmidt A; Pazin Filho A, Maciel BC. Medida indireta da pressão arterial sistêmica. Med 2004;37:240-5.

10. AmooreJN. Oscillometric Sphygmomanometers: A Critical Appraisal of Current Technology. Blood Press Monit 2012;17:80-8.

11. Bordley III J, Connor CAR, Hamilton WF, Kerr WJ, WiggersCJ.Recommendations for Human Blood Pressure Determinations by Sphygmomanometers. Circul 1951;4:503-9.
12. O 'Brien E, Pickering $T$, Asmar Roland, Myers M, Parati G, Staessen J, Mengden T, lami Y, Waeber B, Palatini P. Working Group on Blood Pressure Monitoring of the European Society Hypertension International Protocol for validation of blood pressure measuring devices in adults. Blood Press Monit 2002;7:3-17.

13. Gusmão JL, Cavagioni LC, Colósimo FC, Silva SSBE, Serafim T, Toma GA et al. Os esfigmomanômetros de coluna de mercúrio devem ser eliminados daprática clínica? Rev Bras Hipertens 2008;11(1):20-6.

14. Arcuri EAM. Estudo comparativo da medida indireta da pressão arterial com manguito de largura correta e com manguito de largura padrão. São Paulo. Tese [Doutorado]Universidade de São Paulo; 1985. p. 174.

15. Rabello CCP, Pierin AMG, Mion Jr. D. O conhecimento de profissionais da área da saúde sobre a medida da pressão arterial. Rev Esc Enferm USP 2004;38(2):127-34.

16. Almeida TCF. Enfermeiros de Unidade de Terapia Intensiva Adulto: Conhecimento sobre medida da pressão arterial. São Paulo. Dissertação [Mestrado em Enfermagem]Universidade Estadual de Campinas; 2011. p. 119.

17. Silva SSBE, Colósimo FC, Pierin AMG. O efeito de intervenções educativas no conhecimento da equipe de enfermagem sobre hipertensão arterial. Rev Esc Enferm USP 2010;44(2):488-96. 